\title{
Slowly oscillating perturbations of periodic Jacobi operators in $l^{2}(\mathbb{N})$
}

by

MARCin MoszyŃski (Warszawa)

\begin{abstract}
We prove that the absolutely continuous part of the periodic Jacobi operator does not change (modulo unitary equivalence) under additive perturbations by compact Jacobi operators with weights and diagonals defined in terms of the Stolz classes of slowly oscillating sequences. This result substantially generalizes many previous results, e.g., the one which can be obtained directly by the abstract trace class perturbation theorem of Kato-Rosenblum. It also generalizes several results concerning perturbations of the discrete (free or periodic) Schrödinger operator. The paper concerns "one-sided" Jacobi operators (i.e. in $l^{2}(\mathbb{N})$ ) and is based on the method of subordinacy. We provide some spectral results for the unperturbed, periodic case, and also an appendix containing some subordination theory tools.
\end{abstract}

0. Introduction. Searching for perturbations of a specific "unperturbed" operator which preserve a certain spectral property of that operator is a popular kind of problem in spectral analysis. In this paper, we consider periodic Jacobi operators in $l^{2}(\mathbb{N})$ as unperturbed operators, and we perturb them by adding other Jacobi operators. The main spectral properties studied relate to the absolutely continuous part of the operator.

Similar problems concerning perturbations of the free or periodic discrete Schrödinger operator $(\mathrm{DSO})$ in $l^{2}(\mathbb{N})$ or $l^{2}(\mathbb{Z})$ by diagonal operators have been studied in many papers (see e.g. [2-4, 13-17, 21-23]; see also the introduction and references to [17]).

A famous abstract result on stability of the absolutely continuous part of an operator under perturbations is the Kato-Rosenblum theorem (see e.g. [20]). It states that the absolutely continuous parts of a self-adjoint operator and of its perturbation by an arbitrary trace class operator are unitarily equivalent. Obviously, this theorem can be directly applied to perturbations by any Jacobi operators with weights $\alpha=\left\{\alpha_{n}\right\}_{n \geq 1}$ and diagonals $\beta=$

2000 Mathematics Subject Classification: 47B36, 47B39, 47B25, 47A55, 47A10, 39A11. Key words and phrases: Jacobi matrix (operator), spectral analysis, absolutely continuous spectrum, absolutely continuous operator, pure point spectrum, periodicity, subordinacy, slowly oscillating sequences, perturbations. 
$\left\{\beta_{n}\right\}_{n \geq 1}$ satisfying

$$
\sum_{n=1}^{\infty}\left|\alpha_{n}\right|, \sum_{n=1}^{\infty}\left|\beta_{n}\right|<\infty
$$

since, as is easy to check, the above condition is equivalent to the trace class property of the perturbing operator. Thus, we can say that perturbations satisfying (0.1) are in some sense "trivial" from the point of view of the abstract perturbation theory.

The weights $\alpha$ and diagonals $\beta$ of the perturbing operator considered in this paper belong mostly to the large class $D$ of slowly oscillating sequences introduced by G. Stolz (see [22]). The only extra assumption is that $\alpha$ and $\beta$ converge to 0 , which is equivalent to the compactness of the perturbing operator. Our main result is that under these assumptions the absolutely continuous part of the perturbed Jacobi operator is unitarily equivalent to the absolutely continuous part of the unperturbed one. In particular, the absolutely continuous spectrum remains unchanged.

Stolz [22] considered a similar perturbation problem. The unperturbed operator was the free DSO, and it was perturbed by a diagonal operator given by a $D$ class sequence $\beta$. The result obtained for this special case was stronger than the result of the present paper, since the compactness assumption was not necessary there. Stolz proved that there exist three regions of $\mathbb{R}$, determined in a simple way by $\lim \sup$ and $\lim \inf$ of $\beta$, such that in the first region the perturbed operator is absolutely continuous, in the second it is pure point, and the third is "a region of uncertainty" which is however contained in the essential spectrum. Similar results for perturbations of periodic DSO are also presented in [22]. Here we consider a more general tridiagonal form of the unperturbed operator, and also of the perturbing operator. It seems that a generalization of our results to noncompact $D$-perturbations, in the style of the above paper of Stolz, would be difficult to obtain.

Both Stolz's paper and ours give stronger results than some of the results for diagonal perturbations of DSO, e.g., those in [3, 4, 23]. For instance, Golinskii and Nevai [4] (see also [17, Th. 1.5 + Rem. 1]) obtained some similar results under the stronger assumptions that

$$
\sum_{n=1}^{\infty}\left|\beta_{n+T}-\beta_{n}\right|<\infty \text { and } \beta_{n} \rightarrow 0
$$

for some $T \in \mathbb{N}$, and the unperturbed operator is a $T$-periodic DSO.

It should be stressed that our methods are similar to those of [22]. In particular, we also use subordination theory (see [12]). Moreover, Stolz's main trick of iterated diagonalization of matrices is, in some sense, also present here (but it remains deeply hidden in the proof of a Levinson type theorem, which is used to prove Criterion 5.10). 
The paper is organized as follows. In Section 1 we introduce the notation and we recall some necessary notions. In Section 2 we study some basic properties of periodic Jacobi operators in $l^{2}(\mathbb{N})$. We do it by combining some elementary methods with subordination theory tools. Perturbations of periodic Jacobi operators are studied in Section 3. In particular, the main result of the paper, Theorem 3.2, is formulated there. The proofs of all the results from Sections 2 and 3 are given in Section 4. The appendix consists of several parts containing a few general results used in this paper; some of them (e.g., Corollary 5.12) may become convenient tools for further studies of spectral properties of Jacobi operators. Some of the results in the appendix were published earlier, and we only recall them for convenience of the reader. Some others have not been published yet, and we give their proofs.

1. Preliminaries and notation. Given real sequences $w=\left\{w_{n}\right\}_{n \geq 1}$ and $q=\left\{q_{n}\right\}_{n \geq 1}$, we denote by $J(w, q)$ the Jacobi operator in the Hilbert space $l^{2}(\mathbb{N})$ determined by the infinite Jacobi matrix

$$
\left(\begin{array}{ccccc}
q_{1} & w_{1} & & & \\
w_{1} & q_{2} & w_{2} & & \\
& w_{2} & q_{3} & w_{3} & \\
& & w_{3} & q_{4} & \ddots \\
& & & \ddots & \ddots
\end{array}\right) .
$$

In this paper we consider mainly bounded $w$ and $q$, and then $J(w, q)$ is the bounded self-adjoint operator defined by

$$
(J(w, q) u)_{n}=w_{n-1} u_{n-1}+q_{n} u_{n}+w_{n} u_{n+1}, \quad u \in l^{2}(\mathbb{N}), n \in \mathbb{N},
$$

(in this paper $\mathbb{N}=\{1,2,3, \ldots\}$ ) with the convention that $w_{j}=u_{j}:=0$ if $j<1$. Without the boundedness assumption, the above formula defines $J(w, q) u$ for $u$ from the domain of $J(w, q)$, which is the usual maximal domain.

Let $\lambda \in \mathbb{R}$. Consider a "generalized eigenvector" for $J(w, q)$ and $\lambda$, i.e. a scalar sequence $u:=\left\{u_{n}\right\}_{n \geq 1}$ satisfying

$$
w_{n-1} u_{n-1}+q_{n} u_{n}+w_{n} u_{n+1}=\lambda u_{n}, \quad n \geq 2 .
$$

The main tool used in this paper is the subordination theory for Jacobi operators, described by Khan and Pearson [12] and developed in many papers (see e.g. $[11,18])$. To apply the subordination theory we shall study asymptotic properties of the above generalized eigenvectors. For this purpose, if $w_{n} \neq 0$ for all $n \in \mathbb{N}$, it is convenient to rewrite the equation (1.2) in the $\mathbb{C}^{2}$ vector form (note that we denote vectors as rows, not columns - see end of this section):

$$
\left(u_{n}, u_{n+1}\right)=B_{n}(\lambda)\left(u_{n-1}, u_{n}\right),
$$


where $B_{n}(\lambda)$ is the $n$th transfer matrix for $J(w, q)$ and $\lambda \in \mathbb{R}$, given by

$$
B_{n}(\lambda):=\left(\begin{array}{cc}
0 & 1 \\
-\frac{w_{n-1}}{w_{n}} & \frac{\lambda-q_{n}}{w_{n}}
\end{array}\right) .
$$

We now recall some spectral terminology and notation used; we stress that in the literature some other terms and notations may be used in the same context.

Let $A$ be a self-adjoint operator in a Hilbert space. We shall use the symbols $\mathcal{H}_{\boldsymbol{A}}(A)$, with being ac, sc, pp, or $G$, where $G$ is a Borel subset of $\mathbb{R}$. For the first three cases this symbol denotes the space of absolute continuity, of singular continuity, and the pure point space (i.e. the closure of the space spanned by all the eigenvectors) of $A$, respectively; and $\mathcal{H}_{G}(A)$ is the range of the spectral projection $E_{G}(A)$.

Similarly, for as above, we denote by $(A)$ the restriction of $A$ to $\mathcal{H}_{\boldsymbol{\Delta}}(A)$. In particular, for the first three cases $(A)_{\mathbf{s}}$ is the so-called absolutely continuous, singular continuous and pure point part of $A$, respectively.

As usual, $\sigma_{\mathrm{ac}}(A), \sigma_{\mathrm{sc}}(A), \sigma_{\mathrm{pp}}(A), \sigma_{\mathrm{ess}}(A), \sigma_{\mathrm{d}}(A)$ denote the absolutely continuous, singular continuous, point, essential, and discrete spectrum of $A$, respectively.

Recall that $A$ is absolutely continuous (resp. pure point) in $G$ iff $\mathcal{H}_{G}(A) \subset$ $\mathcal{H}_{\mathrm{ac}}(A)$ (resp. $\left.\mathcal{H}_{G}(A) \subset \mathcal{H}_{\mathrm{pp}}(A)\right)$. For simplicity we often omit "in $G$ " when $G=\mathbb{R}$. Note that $A$ is absolutely continuous (resp. pure point) in $G$ iff $(A)_{G}$ is absolutely continuous (resp. pure point), as is easy to check. Note as well that when $A$ is absolutely continuous in $G$, it is also often said to have "purely absolutely continuous spectrum in $G$ " in the literature (and similarly for the pure point case).

The operator of multiplication by the identity function in the space $L^{2}(G)$ is denoted by $\boldsymbol{X}_{G}$. The symbol $\sim$ denotes unitary equivalence of operators.

Now let us introduce the notation pertaining to the Stolz classes.

For a sequence $a=\left\{a_{n}\right\}_{n \geq n_{0}}$ with terms in a normed space with the norm $\|\cdot\|$ we denote by $\Delta a$ the discrete derivative of $a$, i.e. $(\Delta a)_{n}=a_{n+1}-a_{n}$ for $n \geq n_{0}$. For $k \in \mathbb{N}$ the Stolz classes $D^{k}$ of slowly oscillating sequences (see [22]) are defined as follows: $a \in D^{k}$ (or $a$ is a $D^{k}$ sequence) iff $a \in l^{\infty}$ and $\Delta^{m} a \in l^{k / m}$ for $m=1, \ldots, k$. Here $\Delta^{m}$ denotes the $m$ th power of $\Delta$ and, as usual, for $1 \leq p<\infty, a \in l^{p}$ iff $\sum_{n=n_{0}}^{\infty}\left\|a_{n}\right\|^{p}<\infty$, and $l^{\infty}$ denotes the set of bounded sequences. We also set

$$
D:=\bigcup_{k \in \mathbb{N}} D^{k}
$$

We use the same symbols $\Delta, l^{p}, D^{k}, D$ for all $n_{0}$ and for all the underlying spaces (mainly $\mathbb{C}$ and spaces of scalar matrices). 
We denote by discr $C$ the discriminant of the characteristic polynomial of a $2 \times 2$ matrix $C$, i.e., $\operatorname{discr} C=(\operatorname{tr} C)^{2}-4 \operatorname{det} C$. We usually identify a $2 \times 2$ matrix with the appropriate linear operator acting in $\mathbb{C}^{2}$. In particular, we write $\mathbb{C}^{2}$ vectors as rows, and for a $2 \times 2$ matrix $A$, we denote by $A\left(x_{1}, x_{2}\right)$ the value of the operator given by $A$ on the vector $\left(x_{1}, x_{2}\right)$, i.e., $A\left(x_{1}, x_{2}\right)=$ $\left(A_{11} x_{1}+A_{12} x_{2}, A_{21} x_{1}+A_{22} x_{2}\right)$.

We use the following notation for product of matrices: $\prod_{k=m}^{n} A_{j}$ equals $A_{n} \ldots A_{m}$ if $n>m$; if $n=m$ it equals $A_{m}$, and if $n<m$ it is $I$.

We denote by $e_{1}, e_{2}$ the canonical base vectors $(1,0),(0,1)$ of $\mathbb{C}^{2}$, respectively.

We use the inequalities $<, \leq,>, \geq$ not only for real numbers, but also for subsets of $\mathbb{R}$. For example, $X<Y$ for $X, Y \subset \mathbb{R}$ means that $x<y$ for any $x \in X$ and $y \in Y$, and similarly for the other relations.

2. Periodic Jacobi operators in $l^{2}(\mathbb{N})$. Some spectral properties of periodic Jacobi operators in $l^{2}(\mathbb{N})$ are similar to the properties of analogous "two-sided" periodic Jacobi operators acting in $l^{2}(\mathbb{Z})$ (see e.g. [23, p. 122] for Floquet's theory in $l^{2}(\mathbb{Z})$, and [19] for another approach). However, specific results for $l^{2}(\mathbb{N})$ are not easy to find in the literature. Therefore we collect some of such spectral results in this section (see also [1, Section 3]).

Fix $T \in \mathbb{N}$ and $T$-periodic real sequences $a=\left\{a_{n}\right\}_{n \geq 1}, b=\left\{b_{n}\right\}_{n \geq 1}$ with $a_{n} \neq 0$, and consider the periodic Jacobi operator $J_{0}:=J(a, b)$.

We give some spectral results for $J_{0}$ that can be obtained using mainly subordination and asymptotic methods.

Denote by $B_{k}^{0}(\lambda)$ the $k$ th transfer matrix for $J_{0}$ and $\lambda \in \mathbb{R}$, given by

$$
B_{k}^{0}(\lambda):=\left(\begin{array}{cc}
0 & 1 \\
-\frac{a_{k-1}}{a_{k}} & \frac{\lambda-b_{k}}{a_{k}}
\end{array}\right) .
$$

Observe that by periodicity the " $T$-group products" of transfer matrices are constant, i.e. for any $n \in \mathbb{N}$,

$$
\prod_{s=2}^{T+1} B_{T n+s}^{0}(\lambda)=\prod_{s=2}^{T+1} B_{s}^{0}(\lambda)=: A(\lambda) .
$$

The matrix function $A(\cdot)$ plays an important role in studying the spectral properties of the operator $J_{0}$. Using $a_{1}=a_{T+1}$ we get

$$
\operatorname{det} A(\lambda)=\frac{a_{1}}{a_{2}} \frac{a_{2}}{a_{3}} \cdots \frac{a_{T}}{a_{T+1}}=1,
$$

but $\operatorname{tr} A(\lambda)$ depends on $\lambda$-it is a polynomial of degree $T$.

Now define

$$
S_{\bowtie}=\{\lambda \in \mathbb{R}:|\operatorname{tr} A(\lambda)| \bowtie 2\},
$$


where $\bowtie$ stands for $<,>,=$, or $\leq$. All these sets are determined by the function $\operatorname{tr} A(\cdot)$, which will be called the spectral polynomial for $J_{0}$. The same sets appear in the $l^{2}(\mathbb{Z})$ case, and we can use the following classical results on their geometry (the gaps and bands decomposition, see e.g. [6, 23]):

- $S_{<}=I_{1} \cup \cdots \cup I_{T}$ where $I_{k}$ are nonempty bounded open intervals such that $I_{k}<I_{k+1}$;

- $\overline{S_{<}}=S_{\leq}=\overline{I_{1}} \cup \cdots \cup \overline{I_{T}} \quad\left(\overline{I_{k}}\right.$ — "bands"); in particular $S_{\leq}$has no isolated points;

- $S_{>}=G_{-} \cup G_{1} \cup \cdots \cup G_{T-1} \cup G_{+}$, where $G_{-}$and $G_{+}$are unbounded open intervals, and $G_{k}$ are bounded open intervals ("gaps", some of them may be empty), such that $G_{-}<\overline{I_{1}}, \overline{I_{T}}<G_{+}$, and when $G_{j} \neq \emptyset$, then $\overline{I_{j}}<G_{j}<\overline{I_{j+1}}$ (and the union of all the above $2 T+1$ intervals is equal to $\mathbb{R}$ ).

The previously mentioned spectral results on $J_{0}$ are collected in the following proposition, which is proved in Section 4.

Proposition 2.1.

(a) $J_{0}$ is absolutely continuous in $S_{\leq}$and pure point in $S_{>}$, and $\sigma_{\mathrm{sc}}\left(J_{0}\right)$ $=\emptyset$;

(b) $\sigma_{\mathrm{ess}}\left(J_{0}\right)=\sigma_{\mathrm{ac}}\left(J_{0}\right)=S_{\leq}$;

(c) $\sigma_{\mathrm{d}}\left(J_{0}\right)=\sigma_{\mathrm{pp}}\left(J_{0}\right)$ and it is a finite subset of $S_{>}$; moreover, if $\lambda$ is an eigenvalue of $J_{0}$, then $Q(\lambda)=0$, where $Q$ is the nonzero polynomial defined by

$$
Q(\lambda):=\left[\operatorname{tr} A(\lambda)-2\left(A_{11}(\lambda)+\frac{\lambda-b_{1}}{a_{1}} A_{12}(\lambda)\right)\right]^{2}-(\operatorname{tr} A(\lambda))^{2}+4 ;
$$

(d) $\left(J_{0}\right)_{S_{\leq}}=\left(J_{0}\right)_{S_{<}}=\left(J_{0}\right)_{\text {ac }} \sim \boldsymbol{X}_{S_{<}}$.

It is worth noting that in contrast to the $l^{2}(\mathbb{Z})$ case, the point spectrum of $J_{0}$ may be nonempty. Below we give a necessary and sufficient condition for it when $T=2$ (for the proof see Section 4 ).

Proposition 2.2. Suppose that $T=2$. If $\left|a_{1}\right| \geq\left|a_{2}\right|$, then $\sigma_{\mathrm{pp}}\left(J_{0}\right)=\emptyset$. If $\left|a_{1}\right|<\left|a_{2}\right|$, then $\sigma_{\mathrm{pp}}\left(J_{0}\right)=\left\{b_{1}\right\}$.

The following example can be easily computed using Propositions 2.1 and 2.2 .

EXAMPLE 2.3. Let $T=2$.

A. If $a_{1}=1, a_{2}=2, b_{1}=b_{2}=0$, then

$$
\sigma_{\mathrm{d}}\left(J_{0}\right)=\{0\}, \quad \sigma_{\text {ess }}\left(J_{0}\right)=[-3 ;-1] \cup[1 ; 3] .
$$

B. If $a_{1}=2, a_{2}=1, b_{1}=1, b_{2}=-1$, then

$$
\sigma_{\mathrm{d}}\left(J_{0}\right)=\emptyset, \quad \sigma_{\mathrm{ess}}\left(J_{0}\right)=[-\sqrt{10} ;-\sqrt{2}] \cup[\sqrt{2} ; \sqrt{10}] .
$$


C. If $a_{1}=1, a_{2}=-1, b_{1}=b_{2}=0$, then

$$
\sigma_{\mathrm{d}}\left(J_{0}\right)=\emptyset, \quad \sigma_{\mathrm{ess}}\left(J_{0}\right)=[-2 ; 2]
$$

(in this case $J_{0}$ is unitarily equivalent to the discrete free Schrödinger operator in $\left.l^{2}(\mathbb{N})\right)$.

REMARKS 2.4. 1. Let $J_{0 \mathbb{Z}}$ denote the analogous periodic Jacobi operator acting in $l^{2}(\mathbb{Z})$ (we extend both periodic sequences periodically onto $\mathbb{Z}$ ). It is known (see e.g. [23]) that $J_{0 \mathbb{Z}}$ is an absolutely continuous operator and $\sigma\left(J_{0 \mathbb{Z}}\right)=S_{\leq}$.

2. It can be easily proved that $J_{0 \mathbb{Z}} \equiv\left(J_{0} \oplus J_{0}^{\prime}\right)+C$, where $J_{0}^{\prime}$ is also a periodic Jacobi operator, "the part of $J_{0 \mathbb{Z}}$ for the negative entries", and $C$ is a 2 -dimensional operator. Moreover, one can show that $J_{0}^{\prime} \sim J_{0}$. Hence, by the previous remark, some abstract methods, e.g., the Weyl theorem on compact perturbations, the Kato-Rosenblum theorem on trace class perturbations, or Glazman's splitting method, allow one to prove that each set $\overline{G_{k}}(k=$ $1, \ldots, T-1)$ contains at most one point of $\sigma_{\mathrm{d}}\left(J_{0}\right)$ (in fact, this point must belong to $G_{k}$, by Proposition 2.1), and that no points of $\sigma_{\mathrm{d}}\left(J_{0}\right)$ are in $G_{ \pm}$.

3 . The equation $Q(\lambda)=0$ gives only a necessary condition for the point spectrum, but this condition has an explicit form. The degree of the polynomial $Q$ can be easily estimated by $2 T-1$, which also estimates the number of eigenvalues of $J$. On the other hand, the above remark gives the much better estimate $T-1$. Heinig's paper [5] provides another polynomial of exact degree $T-1$ which vanishes on $\sigma_{\mathrm{pp}}\left(J_{0}\right)$.

3. Invariance of absolutely continuous spectrum under slowly oscillating perturbations. We shall perturb the periodic Jacobi operator $J_{0}$ by a compact Jacobi operator $P:=J(\alpha, \beta)$, defined by real sequences $\alpha=\left\{\alpha_{n}\right\}_{n \geq 1}$ and $\beta=\left\{\beta_{n}\right\}_{n \geq 1}$. We shall say that the perturbation $P$ is

- slowly oscillating iff $\alpha, \beta$ converge to 0 and $\alpha, \beta \in D$;

- T-slowly oscillating iff $\alpha, \beta$ converge to 0 and $\alpha^{\{j\}}, \beta^{\{j\}} \in D$ for any $j=1, \ldots, T$, where for a sequence $x=\left\{x_{n}\right\}_{n \geq 1}$ and $j=0,1, \ldots$ we write

$$
x^{\{j\}}:=\left\{x_{n T+j}\right\}_{n \geq 1}
$$

(in particular, $a^{\{j\}}$ and $b^{\{j\}}$ are constant sequences equal to $a_{j}, b_{j}$, respectively).

It can be easily seen that the second condition is weaker, i.e. a slowly oscillating $P$ is also $T$-slowly oscillating for any $T$ (here it is not important that $T$ is a period related to $J_{0}$; note also that if $P$ is $T$-slowly oscillating, then $\alpha^{\{j\}}, \beta^{\{j\}} \in D$ not only for $j=1, \ldots, T$, but for any $\left.j=0,1, \ldots\right)$. 
Let $J$ denote the perturbed Jacobi matrix,

$$
J:=J_{0}+P .
$$

We shall say that $J$ is a slowly oscillating (resp. T-slowly oscillating) perturbation of $J_{0}$ when $P$ is slowly oscillating (resp. $T$-slowly oscillating).

EXAMPLE 3.1. By the definition, $J$ is a slowly oscillating perturbation of $J_{0}$ iff $J=J(a+\alpha, b+\beta)$ with some $\alpha, \beta$ converging to 0 and $\alpha, \beta \in D$ (additive perturbation). But if $J=J(a \gamma, b \delta)$ and $\gamma, \delta \in D$ with $\gamma_{n}, \delta_{n} \rightarrow 1$ (multiplicative perturbation), then $J$ is a $T$-slowly oscillating perturbation of $J_{0}$.

The following theorem is the main result of this paper (note that the weaker, " $T$ " perturbation condition is sufficient here).

Theorem 3.2. Suppose that $P$ is T-slowly oscillating. Then

$$
(J)_{\mathrm{ac}} \sim\left(J_{0}\right)_{\mathrm{ac}} \sim \boldsymbol{X}_{S_{<}},
$$

and

(i) $\sigma_{\mathrm{ac}}(J)=\sigma_{\mathrm{ess}}(J)=\sigma_{\mathrm{ac}}\left(J_{0}\right)=S_{\leq}$, in particular, $J$ is pure point in $S_{>}$

(ii) $\sigma_{\mathrm{sc}}(J)=\emptyset$;

(iii) if, moreover,

$$
a_{n}+\alpha_{n} \neq 0, \quad n \in \mathbb{N},
$$

then $J$ is absolutely continuous in $S_{<}$and

Remarks 3.3. 1. Under Golinskii and Nevai's conditions from [4] for the periodic DSO case (see also [17, Th. $1.5+$ Rem. 1]), i.e., if $a_{n}=1$ and $\alpha_{n}=0$ for all $n \in \mathbb{N}$, and (0.2) holds, then all the assumptions of the above theorem hold.

2. Some interesting results on the "discreteness of the point spectrum" can be found in [1].

4. The proofs. In this section we prove Propositions 2.1 and 2.2 , and Theorem 3.2. The main general tools used in these proofs, in particular the class $H$, are recalled in the appendix.

Let us start from some simple observations on the matrix $A(\lambda)$ and the sets $S_{\bowtie}$ :

$$
\lambda \in S_{<}, S_{=}, S_{>} \text {iff } \operatorname{discr} A(\lambda)<0,=0,>0 \text {, respectively. }
$$

This is a consequence of (2.3).

For $\lambda \in \mathbb{R}$ let $\mu_{1}(\lambda), \mu_{2}(\lambda)$ be the eigenvalues of $A(\lambda)$; by the above remark, their ordering can be chosen as indicated below. 
(i) If $\lambda \in S_{<}$, then there are two different mutually conjugate nonreal eigenvalues with absolute value 1 , so we assume $\operatorname{Im} \mu_{1}(\lambda)>0$, $\operatorname{Im} \mu_{2}(\lambda)<0$.

(ii) If $\lambda \in S_{=}$, then $\mu_{1}(\lambda)=\mu_{2}(\lambda)= \pm 1$.

(iii) If $\lambda \in S_{>}$, then there are two different real eigenvalues with product 1 , so we assume

$$
0<\left|\mu_{1}(\lambda)\right|<1<\left|\mu_{2}(\lambda)\right| .
$$

We now prove the following result on the eigenvalues of $J_{0}$.

Lemma 4.1. Suppose that $\lambda \in \mathbb{R}$, and set $p(\lambda):=\left\{p_{n}(\lambda)\right\}_{n \geq 0}$ where $p_{n}(\lambda)$ are the $\mathbb{C}^{2}$ vectors given by

$$
p_{n}(\lambda):=(A(\lambda))^{n}\left(1, \frac{\lambda-b_{1}}{a_{1}}\right) .
$$

(a) $\lambda \in \sigma_{\mathrm{pp}}\left(J_{0}\right)$ iff $p(\lambda) \in l^{2}$.

(b) Suppose that $\lambda \in S_{>}$. Then $\lambda \in \sigma_{\mathrm{pp}}\left(J_{0}\right)$ iff

$$
\left(A(\lambda)-\mu_{1}(\lambda) I\right)\left(1, \frac{\lambda-b_{1}}{a_{1}}\right)=0 .
$$

In particular, in this case, if $\lambda \in \sigma_{\mathrm{pp}}\left(J_{0}\right)$, then $Q(\lambda)=0$, where $Q$ is the nonzero polynomial defined by (2.5).

(c) If $\lambda \in S_{\leq}$, then $\lambda \notin \sigma_{\mathrm{pp}}\left(J_{0}\right)$.

Proof. Consider a generalized eigenvector $u(\lambda):=\left\{u_{n}(\lambda)\right\}_{n \geq 1}$ satisfying

$$
a_{n-1} u_{n-1}(\lambda)+b_{n} u_{n}(\lambda)+a_{n} u_{n+1}(\lambda)=\lambda u_{n}(\lambda), \quad n \geq 2,
$$

and

$$
u_{1}(\lambda)=1, \quad u_{2}(\lambda)=\frac{\lambda-b_{1}}{a_{1}} .
$$

Obviously, $\lambda \in \sigma_{\mathrm{pp}}\left(J_{0}\right)$ iff $u(\lambda) \in l^{2}$. Writing (1.2) in the $\mathbb{C}^{2}$ vector form

$$
\left(u_{n}(\lambda), u_{n+1}(\lambda)\right)=B_{n}^{0}(\lambda)\left(u_{n-1}(\lambda), u_{n}(\lambda)\right),
$$

and using $(2.2)$ we get

$$
p_{n}(\lambda)=\left(u_{n T+1}(\lambda), u_{n T+2}(\lambda)\right) .
$$

Thus, if $u(\lambda) \in l^{2}$ then $p(\lambda) \in l^{2}$. On the other hand, if $p(\lambda) \in l^{2}$ then by (4.5), (4.6) and by the invertibility of the matrices $B_{s}^{0}(\lambda), s=2, \ldots, T+1$, also $u(\lambda) \in l^{2}$. This proves assertion (a).

Assume now that $\lambda \in S_{>}$. By (4.1) we can diagonalize $A(\lambda)$,

$$
A(\lambda)=Y\left(\begin{array}{cc}
\mu_{1}(\lambda) & 0 \\
0 & \mu_{2}(\lambda)
\end{array}\right) Y^{-1},
$$

and by (4.2) we obtain

$$
p_{n}(\lambda):=Y\left(c_{1}\left(\mu_{1}(\lambda)\right)^{n}, c_{2}\left(\mu_{2}(\lambda)\right)^{n}\right),
$$


with some invertible ( $\lambda$-dependent) matrix $Y$ and with $c:=\left(c_{1}, c_{2}\right)=$ $Y^{-1}\left(1,\left(\lambda-b_{1}\right) / a_{1}\right)$. Thus by $(4.1)$, it follows that $p(\lambda) \in l^{2}$ iff $c_{2}=0$ iff $\left(\begin{array}{cc}\mu_{1}(\lambda) & 0 \\ 0 & \mu_{2}(\lambda)\end{array}\right)=\mu_{1}(\lambda) c$ iff (4.3) holds. Hence, by (a), to finish the proof of (b) it remains to prove that $Q$ is nonzero, and that $Q(\lambda)=0$ if (4.3) is satisfied. By the definition of $\mu_{1}(\lambda)$ we have

$$
\mu_{1}(\lambda)=\frac{1}{2}\left[\sigma(\lambda) \sqrt{(\operatorname{tr} A(\lambda))^{2}-4}+\operatorname{tr} A(\lambda)\right],
$$

where $\sigma(\lambda)$ is 1 or -1 . Suppose (4.3) holds. Thus in particular

$$
A_{11}(\lambda)-\mu_{1}(\lambda)+A_{12}(\lambda) \frac{\lambda-b_{1}}{a_{1}}=0,
$$

and using (4.7) we get

$$
2 A_{11}(\lambda)+2 A_{12}(\lambda) \frac{\lambda-b_{1}}{a_{1}}-\operatorname{tr} A(\lambda)=\sigma \sqrt{(\operatorname{tr} A(\lambda))^{2}-4},
$$

which immediately gives $Q(\lambda)=0$.

Suppose now that $Q$ is a zero polynomial. Defining

$$
f_{1}(\lambda):=\operatorname{tr} A(\lambda)-2\left(A_{11}(\lambda)+\frac{\lambda-b_{1}}{a_{1}} A_{12}(\lambda)\right), \quad f_{2}(\lambda):=\operatorname{tr} A(\lambda),
$$

we obtain $f_{1}^{2}-f_{2}^{2}=\left(f_{1}-f_{2}\right)\left(f_{1}+f_{2}\right)=-4$, so both $f_{1}-f_{2}$ and $f_{1}+f_{2}$ must be constant functions. Therefore also both $f_{1}$ and $f_{2}$ must be constant, which is a contradiction, since $f_{2}$ is a polynomial of degree $T$.

To prove (c) assume first that $\lambda \in S_{<}$. In this case $A(\lambda)$ is also diagonalizable, so just as in the case $\lambda \in S_{>}$, for some invertible matrix $Y$ we obtain

$$
p_{n}(\lambda):=Y\left(c_{1}\left(\mu_{1}(\lambda)\right)^{n}, c_{2}\left(\mu_{2}(\lambda)\right)^{n}\right)
$$

with $c:=\left(c_{1}, c_{2}\right)=Y^{-1}\left(1,\left(\lambda-b_{1}\right) / a_{1}\right)$. Hence $p(\lambda) \notin l^{2}$, because $c \neq 0$ and $\left.\left|\mu_{1}(\lambda)\right|=\mid \mu_{(} \lambda\right) \mid=1$. Thus by (a), $\lambda \notin \sigma_{\mathrm{pp}}\left(J_{0}\right)$.

If $\lambda \in S_{=}$, then there are two possibilities. If $A(\lambda)$ is diagonalizable, then we can repeat the above argument. If $A(\lambda)$ is not diagonalizable, then it is similar to a Jordan cell, i.e. for some invertible matrix $Y$,

$$
A(\lambda)=Y\left(\begin{array}{cc}
z & 1 \\
0 & z
\end{array}\right) Y^{-1}, \quad(A(\lambda))^{n}=Y\left(\begin{array}{cc}
z^{n} & n z^{n-1} \\
0 & z^{n}
\end{array}\right) Y^{-1}, \quad n \in \mathbb{N},
$$

where $z= \pm 1$. Combining this with (4.2) yields

$$
p_{n}(\lambda):=Y\left(c_{1} z^{n}+c_{2} n z^{n-1}, c_{2} z^{n}\right)
$$

with $c \neq 0$ (as above), and this obviously also excludes $p(\lambda) \in l^{2}$.

Proof of Proposition 2.1. By (2.2), using Proposition 5.7 and Criterion 5.8 , we see that $\left\{B_{n}^{0}\right\}_{n \geq 2} \in H$, provided discr $A(\lambda)<0$. Hence, $\left\{B_{n}^{0}\right\}_{n \geq 2} \in H$ for any $\lambda \in S_{<}$. Moreover, $S_{<}$is an open set. Theorem 5.6 now implies that $J_{0}$ is absolutely continuous in $S_{<},\left(J_{0}\right)_{S_{<}} \sim \boldsymbol{X}_{S_{<}}$and $\overline{S_{<}} \subset \sigma_{\mathrm{ac}}\left(J_{0}\right)$. 
Let now $\lambda \in S_{>}$and consider the equation

$$
x_{n+1}=C_{n}^{0}(\lambda) x_{n}, \quad n \geq 0,
$$

where $x=\left\{x_{n}\right\}_{n \geq 0}$ is a sequence of $\mathbb{C}^{2}$ vectors, and

$$
C_{n}^{0}(\lambda):=\prod_{s=2}^{T+1} B_{T n+s}^{0}(\lambda), \quad n \geq 0 .
$$

By (2.2) all solutions of (4.8) are given by

$$
x_{n}:=(A(\lambda))^{n} x_{0},
$$

where the vector $x_{0}$ can be arbitrary. Diagonalizing $A(\lambda)$ as in the proof of Lemma 4.1 we obtain

$$
x_{n}:=Y\left(\begin{array}{cc}
\left(\mu_{1}(\lambda)\right)^{n} & 0 \\
0 & \left(\mu_{2}(\lambda)\right)^{n}
\end{array}\right) Y^{-1} x_{0}
$$

with some invertible matrix $Y$. Taking $x_{0}:=Y(1,0)$ we get $x_{n}=\mu_{1}^{n} x_{0}$, hence such a solution $x$ of (4.8) is in $l^{2}$, by (4.1). Therefore, by Theorem $5.14, J_{0}$ is pure point in $S_{>}$.

Lemma 4.1 shows that $\sigma_{\mathrm{pp}}\left(J_{0}\right)$ is a finite subset of $S_{>}$(and the multiplicity of each eigenvalue is one, which is a standard fact for Jacobi operators). In particular, $E_{\{\lambda\}}\left(J_{0}\right)=0$ for any $\lambda \in S_{\leq}$. Since $S_{=}$is a finite subset of $S_{\leq}$, we have $E_{S_{=}}\left(J_{0}\right)=0$, and consequently $\mathcal{H}_{S_{\leq}}\left(J_{0}\right)=\mathcal{H}_{S_{<}}\left(J_{0}\right)$. This implies that $J_{0}$ is absolutely continuous also in $S_{\leq}$, and that $\left(J_{0}\right)_{S_{\leq}}=\left(J_{0}\right)_{S_{<}}$.

Above we have proved that $\mathcal{H}_{S_{\leq}}\left(J_{0}\right) \subset \mathcal{H}_{\mathrm{ac}}\left(J_{0}\right)$ and $\mathcal{H}_{S_{>}}\left(J_{0}\right) \subset \mathcal{H}_{\mathrm{pp}}\left(J_{0}\right)$. Hence, using the fact that

$$
\mathcal{H}_{S_{\leq}}\left(J_{0}\right)+\mathcal{H}_{S_{>}}\left(J_{0}\right)=l^{2}(\mathbb{N})
$$

(because $S_{\leq} \cup S_{>}=\mathbb{R}$ ), and that

$$
\mathcal{H}_{\mathrm{ac}}\left(J_{0}\right) \oplus \mathcal{H}_{\mathrm{pp}}\left(J_{0}\right) \oplus \mathcal{H}_{\mathrm{sc}}\left(J_{0}\right)=l^{2}(\mathbb{N}),
$$

we get $\mathcal{H}_{\mathrm{sc}}\left(J_{0}\right)=\{0\}, \mathcal{H}_{S_{\leq}}\left(J_{0}\right)=\mathcal{H}_{\mathrm{ac}}\left(J_{0}\right)$, and $\mathcal{H}_{S_{>}}\left(J_{0}\right)=\mathcal{H}_{\mathrm{pp}}\left(J_{0}\right)$. Thus $\left(J_{0}\right)_{S_{<}}=\left(J_{0}\right)_{S_{\leq}}=\left(J_{0}\right)_{\text {ac }}$ (which finishes the proof of $\left.(\mathrm{d})\right)$, and this gives $\sigma_{\mathrm{ac}}\left(J_{0}\right)=\sigma\left(\left(J_{0}\right)_{\mathrm{ac}}\right)=\sigma\left(\left(J_{0}\right)_{S_{<}}\right) \subset \overline{S_{<}}$, the inclusion being a consequence of Proposition 5.15. By the opposite inclusion (already proved) we get $\sigma_{\text {ac }}\left(J_{0}\right)=$ $\overline{S_{<}}=S_{\leq}$. Moreover, we have $\sigma_{\mathrm{sc}}\left(J_{0}\right)=\emptyset$ (which finishes the proof of (a)), and thus $\sigma\left(J_{0}\right)=S_{\leq} \cup \overline{\sigma_{\mathrm{pp}}\left(J_{0}\right)}$. Hence, knowing that $\sigma_{\mathrm{pp}}\left(J_{0}\right)$ is a finite subset of $S_{>}$(recall also that each eigenvalue is simple), and that $S_{>}$is an open set disjoint from $S_{\leq}$, we immediately conclude that $\sigma_{\mathrm{d}}\left(J_{0}\right)=\sigma_{\mathrm{pp}}\left(J_{0}\right)$ and $\sigma_{\text {ess }}\left(J_{0}\right)=S_{\leq}$; this finishes the proof of (b) and begins the proof of (c). The rest of the assertion of (c) is directly formulated in Lemma 4.1. 
Proof of Proposition 2.2. If $T=2$, then by (2.2),

$$
A(\lambda)=\left(\begin{array}{cc}
-\frac{a_{1}}{a_{2}} & \frac{\lambda-b_{2}}{a_{2}} \\
\frac{b_{1}-\lambda}{a_{2}} & \frac{\left(\lambda-b_{1}\right)\left(\lambda-b_{2}\right)}{a_{1} a_{2}}-\frac{a_{2}}{a_{1}}
\end{array}\right)
$$

and

$$
\operatorname{tr} A(\lambda)=\left(a_{1} a_{2}\right)^{-1}\left[\left(\lambda-b_{1}\right)\left(\lambda-b_{2}\right)-\left(a_{1}^{2}+a_{2}^{2}\right)\right] .
$$

The polynomial $Q$ is given by

$$
Q(\lambda)=4+4 a_{1}^{-2}\left[\left(\lambda-b_{1}\right)\left(\lambda-b_{2}\right)-a_{1}^{2}\right] .
$$

Thus the equation $Q(\lambda)=0$ has the solutions $\lambda=b_{1}, b_{2}$, and $\sigma_{\mathrm{pp}}\left(J_{0}\right) \subset$ $\left\{b_{1}, b_{2}\right\}$ by Lemma $4.1(\mathrm{~b})$, (c). So, let now $\lambda \in\left\{b_{1}, b_{2}\right\}$. By (4.9) the matrix $A(\lambda)$ is triangular, and thus its eigenvalue $\mu_{1}(\lambda)$ is one of the diagonal entries $-a_{1} / a_{2},-a_{2} / a_{1}$. Set

$$
y(\lambda):=\left(A(\lambda)-\mu_{1}(\lambda) I\right)\left(1, \frac{\lambda-b_{1}}{a_{1}}\right) .
$$

By Lemma 4.1(b), $\lambda \in \sigma_{\mathrm{pp}}\left(J_{0}\right)$ iff (4.3) holds, i.e., iff $y(\lambda)=0$.

Consider the following cases:

(i) $\left|a_{1}\right|>\left|a_{2}\right|$. Then $\mu_{1}(\lambda)=-a_{2} / a_{1}$, and $y_{1}(\lambda)=a_{2} / a_{1}-a_{1} / a_{2}$. So, $y_{1}(\lambda) \neq 0$ in this case, which proves that $\sigma_{\mathrm{pp}}\left(J_{0}\right)=\emptyset$.

(ii) $\left|a_{1}\right|=\left|a_{2}\right|$. Then $|\operatorname{tr} A(\lambda)|=\left|a_{1} a_{2}\right|^{-1}\left(a_{1}^{2}+a_{2}^{2}\right)=2$, and thus $\lambda \notin S_{>}$. Therefore Lemma 4.1(c) gives $\sigma_{\mathrm{pp}}\left(J_{0}\right)=\emptyset$.

(iii) $\left|a_{1}\right|<\left|a_{2}\right|$. Then $\mu_{1}(\lambda)=-a_{1} / a_{2}$. We have $y\left(b_{1}\right)=0$, hence $b_{1} \in$ $\sigma_{\mathrm{pp}}\left(J_{0}\right)$. If $b_{2} \neq b_{1}$, then

$$
y_{2}\left(b_{2}\right)=\left(b_{1}-b_{2}\right) \frac{a_{2}}{a_{1}^{2}} \neq 0,
$$

which yields $\sigma_{\mathrm{pp}}\left(J_{0}\right)=\left\{b_{1}\right\}$.

Proof of Theorem 3.2. Assume first (3.2). Similarly to the unperturbed case, define the " $T$-group products" of transfer matrices for $J$ :

$$
C_{n}(\lambda):=\prod_{s=2}^{T+1} B_{T n+s}(\lambda), \quad n \geq 0
$$

where now

$$
B_{k}(\lambda):=\left(\begin{array}{cc}
0 & 1 \\
-\frac{a_{k-1}+\alpha_{k-1}}{a_{k}+\alpha_{k}} & \frac{\lambda-\left(b_{k}+\beta_{k}\right)}{a_{k}+\alpha_{k}}
\end{array}\right), \quad k \geq 2 .
$$

We have

$$
B_{T n+j}(\lambda)=\left(\begin{array}{cc}
0 & 1 \\
-\frac{a_{j-1}+\alpha_{n}^{\{j-1\}}}{a_{j}+\alpha_{n}^{\{j\}}} & \frac{\lambda-\left(b_{j}+\alpha_{n}^{\{j\}}\right)}{a_{j}+\alpha_{n}^{\{j\}}}
\end{array}\right) .
$$


Since $P$ is $T$-slowly oscillating, Proposition 5.2(ii) shows that $\left\{B_{T n+j}(\lambda)\right\}_{n \geq 0}$ $\in D$ for any fixed $j$ and $\lambda$. Hence, by (4.10), $\left\{C_{n}(\lambda)\right\}_{n \geq 0} \in D$, since the class $D$ is an algebra (see Appendix 5.1). We also have $B_{T n+j}(\lambda) \rightarrow B_{j}^{0}(\lambda)$, and so by (4.10) and (2.2) we get $C_{n}(\lambda) \rightarrow A(\lambda)$. By (3.2) and Theorem 5.12 we deduce that $J$ is absolutely continuous in $S_{<},(J)_{S_{<}} \sim \boldsymbol{X}_{S_{<}}$and $\overline{S_{<}}=S_{\leq} \subset \sigma_{\text {ac }}(J)$. By Proposition 2.1 this proves (3.1).

As $\bar{J}$ is a compact perturbation of $J_{0}$, by the Weyl theorem and by Proposition 2.1 we have $\sigma_{\text {ess }}(J)=\sigma_{\text {ess }}\left(J_{0}\right)=\sigma_{\text {ac }}\left(J_{0}\right)=S_{\leq}$. Thus

$$
S_{\leq}=\sigma_{\text {ess }}(J) \supset \sigma_{\text {ac }}(J) \supset S_{\leq},
$$

which gives $\sigma_{\text {ac }}(J)=\sigma_{\text {ess }}(J)$. To see that $J$ is pure point in $S_{>}$, observe first that $S_{>}=\sigma_{\mathrm{d}}(J) \cup U$, where $U$ is an open set disjoint from $\sigma(J)$. Hence $\mathcal{H}_{S_{>}}(J)=\mathcal{H}_{\sigma_{\mathrm{d}}(J)}(J) \subset \mathcal{H}_{\mathrm{pp}}(J)$. So we have just proved (i). Thus we can write $\sigma(J)=S_{<} \cup Z$, where $Z=S_{=} \cup \sigma_{\mathrm{d}}(J)$, so $Z$ is an at most countable set disjoint from $S_{<}$. Hence $\mathcal{H}_{S_{<}}(J) \oplus \mathcal{H}_{Z}(J)=l^{2}(\mathbb{N})$. By Proposition 5.15, $\mathcal{H}_{Z}(J) \subset \mathcal{H}_{\mathrm{pp}}(J)$, and above we have proved that $\mathcal{H}_{S_{<}}(J) \subset \mathcal{H}_{\mathrm{ac}}(J)$. Now, since

$$
\mathcal{H}_{\mathrm{ac}}(J) \oplus \mathcal{H}_{\mathrm{pp}}(J) \oplus \mathcal{H}_{\mathrm{sc}}(J)=l^{2}(\mathbb{N})
$$

we get $\mathcal{H}_{\mathrm{sc}}(J)=\{0\}, \mathcal{H}_{S_{<}}(J)=\mathcal{H}_{\mathrm{ac}}(J)$, and $\mathcal{H}_{Z}(J)=\mathcal{H}_{\mathrm{pp}}(J)$. Thus $(J)_{S_{<}}=(J)_{\text {ac }}$ and $\sigma_{\mathrm{sc}}\left(J_{0}\right)=\emptyset$, i.e. (ii) and (iii) hold. This finishes the proof of the theorem under the extra assumption (3.2).

Suppose now that (3.2) does not hold. Then there exists $N \in \mathbb{N}$ such that

$$
a_{N}+\alpha_{N}=0
$$

and $a_{n}+\alpha_{n} \neq 0$ for $n>N$. Define $a^{\prime}, b^{\prime}, \alpha^{\prime}, \beta^{\prime}$ by

$$
a_{n}^{\prime}=a_{n+N}, b_{n}^{\prime}=b_{n+N}, \quad \alpha_{n}^{\prime}=\alpha_{n+N}, \beta_{n}^{\prime}=\beta_{n+N}, \quad n \geq 1 .
$$

Thus we can apply the part already proved to the perturbation of the operator $J_{0}^{\prime}:=J\left(a^{\prime}, b^{\prime}\right)$ by $P^{\prime}:=J\left(\alpha^{\prime}, \beta^{\prime}\right)$. By (4.13), $J$ is unitarily equivalent to the direct orthogonal sum $J_{\text {fin }} \oplus J^{\prime}$, where $J_{\text {fin }}$ is an operator acting in $\mathbb{C}^{N}$. Moreover, the spectral polynomial for $J_{0}^{\prime}$ is equal to the spectral polynomial of $J_{0}$ (use the identity $\operatorname{tr}(X Y)=\operatorname{tr}(Y X)$ ). This gives the equality of all the relevant sets for both periodic Jacobi operators, which finishes the proof.

\section{Appendix}

5.1. The $D$ class. The classes $D^{k}, D$ (see introduction for the definitions) and some related classes have been introduced by Stolz in [22].

Note that $D^{1}$ is the well-known class of bounded variation sequences (thus, e.g., the scalar sequences $\left\{1 / n^{c}\right\}_{n \geq 1}$ are in $D^{1}$ for any $c \geq 0$ ), in particular $D^{1}$ sequences in a Banach space $X$ are always convergent, which is not true for $D^{k}$ sequences with $k>1$. We have $D^{k} \subset D^{k+1} \subset D$. A large 
class of scalar $D^{k}$ sequences can be constructed with the use of the following criterion (see $[22]$ ).

Proposition 5.1. Let $x=\left\{x_{n}\right\}_{n \geq n_{0}}$ be a scalar sequence given by $x_{n}=$ $f(n)$, where $f:\left[n_{0} ; \infty\right) \rightarrow \mathbb{C}$ is $k$ times differentiable with

$$
\partial^{j} f \in L^{k / j}\left(\left[n_{0} ; \infty\right)\right), \quad j=1, \ldots, k .
$$

Then $x \in D^{k}$.

In particular, $\left\{h\left(n^{\gamma}\right)\right\}_{n \geq n_{0}} \in D$ for any $\gamma \in(0 ; 1)$ and every $C^{\infty}$ function $h$ such that its derivatives of all orders are bounded (e.g. for $h$ being sin or cos). Obviously, a matrix sequence is in $D^{k}$ iff all its matrix element sequences are in $D^{k}$.

The scalar and matrix $D^{k}$ classes are algebras with the usual multiplication. Moreover, as seen from the proposition below, many other operations on $D^{k}$ sequences preserve the class $D^{k}$ (choosing properly the function $f$ below, we can also cover multivariable operations).

\section{PROPOSITION 5.2.}

(i) Suppose that $X, X^{\prime}$ are finite-dimensional real normed spaces and that $K \subset U \subset X$, where $U$ is open, and $K$ is compact and convex. If $f: U \rightarrow X^{\prime}$ is a $C^{k}$ function and $x$ is a $D^{k}$ sequence in $K$, then $f(x):=\left\{f\left(x_{n}\right)\right\}_{n \geq n_{0}}$ is a $D^{k}$ sequence in $X^{\prime}$.

(ii) Suppose that $x:=\left\{x_{n}\right\}_{n \geq n_{0}}$ and $y:=\left\{y_{n}\right\}_{n \geq n_{0}}$ are two scalar $D^{k}$ sequences and that $\inf _{n \geq n_{0}}\left|y_{n}\right|>0$. Then $x / y \in D^{k}$.

The proof of the above result for some generalizations of $D^{k}$ classes (the so-called weighted $D^{k}$ classes) can be found in [9].

5.2. The class $H$ and absolute continuity. In this section, and also in the next one, we consider a Jacobi operator $J=J(w, q)$ (not necessarily bounded), with $w_{n}, q_{n} \in \mathbb{R}$. We usually also assume that

$$
w_{n} \neq 0, \quad n \in \mathbb{N} .
$$

We study relations between properties of the sequence of transfer matrices $B_{n}(\lambda)$ for $J$ (see (1.4)) and the absolute continuity of $J$ in some subsets of $\mathbb{R}$.

Let us recall the definition and some properties of a class $H$ of matrix sequences, introduced in [18]. It is a convenient tool in the study of the absolutely continuous part of some Jacobi operators. We recall the appropriate spectral result, and we prove some new ones.

Consider a sequence $\left\{C_{n}\right\}_{n \geq n_{0}}$ of complex $2 \times 2$ matrices. Below we use $\|\cdot\|$ for the usual operator norm for matrices, and $\|\cdot\|_{\text {sp }}$ for the spectral "norm". 
Definition 5.3. $\left\{C_{n}\right\}_{n \geq n_{0}} \in H$ iff there exists $M>0$ such that

$$
\left\|\prod_{k=n_{0}}^{n} C_{k}\right\|^{2} \leq M \prod_{k=n_{0}}^{n}\left|\operatorname{det} C_{k}\right|, \quad n \geq n_{0} .
$$

REMARK 5.4. Let $P_{n}:=\prod_{k=n_{0}}^{n} C_{k}$ and suppose that $\sigma\left(P_{n}\right)=\left\{\mu_{n}^{(1)}, \mu_{n}^{(2)}\right\}$ with $\left|\mu_{n}^{(1)}\right| \leq\left|\mu_{n}^{(2)}\right|$. Then

$$
\left\|\prod_{k=n_{0}}^{n} C_{k}\right\|^{2} \geq\left\|P_{n}\right\|_{\mathrm{sp}}^{2}=\left|\mu_{n}^{(2)}\right|^{2} \geq\left|\mu_{n}^{(1)} \mu_{n}^{(2)}\right|=\left|\operatorname{det} P_{n}\right|=\prod_{k=n_{0}}^{n}\left|\operatorname{det} C_{k}\right|,
$$

which shows that the inequality in the definition of $H$ is a strong condition, since above we have obtained the opposite inequality with $M=1$.

The first reason to apply the class $H$ in the study of spectral properties of Jacobi operators is the following:

Proposition 5.5. If (5.1) holds and the sequence $\left\{B_{n}(\lambda)\right\}_{n \geq 2}$ of transfer matrices for $J$ and $\lambda$ is in $H$, then for any solution $u$ of (1.2),

$$
\exists_{M \in \mathbb{R}} \forall_{n \in \mathbb{N}} \quad \sum_{k=1}^{n+1}\left|u_{k}\right|^{2} \leq M \sum_{k=1}^{n} \frac{1}{\left|w_{k}\right|} .
$$

In particular, (1.2) has no subordinated solutions.

Recall that the first assertion of the above proposition is exactly the socalled generalized Behncke-Stolz condition for $J$ and $\lambda$ introduced by Janas and Naboko as the assumption of the Generalized Behncke-Stolz Lemma (for short, GBS; see e.g. [7, Lemma 1.5]).

We omit the proof of Proposition 5.5 because the proof of the first part is in fact contained in the short proof of Theorem 1.2 of [18], and the second part follows just from GBS.

Some spectral consequences of the generalized Behncke-Stolz condition, which follow from GBS and from subordination theory [12], have been formulated in Theorem 1.1 of [18]. The theorem below combines this result (see also [18, Theorem 1.2]) with Proposition 5.5.

THeOREM 5.6. Suppose that $J$ is self-adjoint, and that (5.1) holds. If $G$ is an open subset of $\mathbb{R}$ and $\left\{B_{n}(\lambda)\right\}_{n \geq 2} \in H$ for any $\lambda \in G$, then $(J)_{G} \sim \boldsymbol{X}_{G}$, $J$ is absolutely continuous in $G$ and $\bar{G} \subset \sigma_{\mathrm{ac}}(J)$.

The second reason for using the class $H$ is that it is fairly easy to check that a sequence of matrices belongs to $H$ (despite Remark 5.4) in many important cases, because there exist several sufficient criteria for membership in $H$.

Let us first mention the following two easy properties of $H$. 
Proposition 5.7. Suppose that $C_{n}$ are invertible matrices for any $n \geq n_{0}$.

(i) If $n_{1} \geq n_{0}$, then $\left\{C_{n}\right\}_{n \geq n_{0}} \in H$ iff $\left\{C_{n}\right\}_{n \geq n_{1}} \in H$.

(ii) If $\left\{C_{n}\right\}_{n \geq n_{0}}$ and $\left\{C_{n}^{-1}\right\}_{n \geq n_{0}}$ are bounded, $T \in \mathbb{N}$ and $l \in\{0,1, \ldots, T\}$, then $\left\{C_{n}\right\}_{n \geq n_{0}} \in H$ iff $\left\{\prod_{s=0}^{T-1} C_{T n+l+s}\right\}_{n \geq n_{0}} \in H$.

A simple criterion guaranteeing that two classes of sequences of matrices are in $H$ has been proved in [18]. It is based on some $D^{1}$ (i.e., bounded variation) assumptions.

Criterion 5.8.

(1) If $C_{n}$ are real invertible complex matrices for $n \geq n_{0}$ and $\left\{C_{n}\right\}_{n \geq n_{0}}$ is a $D^{1}$ sequence with $C_{n} \rightarrow C$, and $\operatorname{discr} C<0$, then $\left\{C_{n}\right\}_{n \geq n_{0}} \in H$.

(2) Suppose that $C_{n}$ are invertible matrices, and $C_{n}$ has the form $C_{n}=$ $a_{n} I+p_{n} S_{n}+R_{n}, n \geq n_{0}$, where

(i) $a_{n}, p_{n} \in \mathbb{R}$ for $n \geq n_{0}, a_{n} \rightarrow a \in \mathbb{R} \backslash\{0\}$ and $p_{n} \rightarrow 0$;

(ii) $\left\{S_{n}\right\}_{n \geq n_{0}}$ is a real $D^{1}$ sequence and $S_{n} \rightarrow S$ with $\operatorname{discr} S<0$;

(iii) $\left\{R_{n}\right\}_{n \geq n_{0}} \in l^{1}$.

Then $\left\{C_{n}\right\}_{n \geq n_{0}} \in H$.

We shall prove a generalization of Criterion 5.8(1) for general $D$ sequences. The proof will use the following lemma. Below we use ${ }^{\top}$ to denote transposition of matrices, in particular, for $v$ a $\mathbb{C}^{2}$ vector, $v^{\top}$ denotes the corresponding $\mathbb{C}^{2}$ column.

Consider the equation

$$
x_{n+1}=C_{n} x_{n}, \quad n \geq n_{0},
$$

for a sequence $x=\left\{x_{n}\right\}_{n \geq n_{0}}$ of $\mathbb{C}^{2}$ vectors.

Lemma 5.9. Suppose that (5.3) has two solutions $x^{1}, x^{2}$ of the form

$$
x_{n}^{m}=\varphi_{n}^{m} y_{n}^{m}, \quad n \geq n_{0}, m=1,2,
$$

where $y^{m}=\left\{y_{n}^{m}\right\}_{n \geq n_{0}}$ are sequences of $\mathbb{C}^{2}$ vectors such that

$$
\sup _{n \geq n_{0}}\left\|y_{n}^{m}\right\|<\infty
$$

for $m=1,2$,

$$
\inf _{n \geq n_{0}}\left|\operatorname{det}\left(\left(y_{n}^{1}\right)^{\top},\left(y_{n}^{2}\right)^{\top}\right)\right|>0,
$$

and $\varphi^{m}=\left\{\varphi_{n}^{m}\right\}_{n \geq n_{0}}$ are sequences of nonzero numbers satisfying

$$
\inf _{n \geq n_{0}}\left|\frac{\varphi_{n}^{1}}{\varphi_{n}^{2}}\right|>0, \quad \sup _{n \geq n_{0}}\left|\frac{\varphi_{n}^{1}}{\varphi_{n}^{2}}\right|<\infty .
$$

Then $\left\{C_{n}\right\}_{n \geq n_{0}} \in H$. 
Proof. Let $X_{n}:=\left(\left(x_{n}^{1}\right)^{\top},\left(x_{n}^{2}\right)^{\top}\right)$. Thus $X_{n+1}=C_{n} X_{n}$ for $n \geq n_{0}$, which gives

$$
\prod_{k=n_{0}}^{n} C_{k}=X_{n+1}\left(X_{n_{0}}\right)^{-1}=\left(\left(y_{n+1}^{1}\right)^{\top},\left(y_{n+1}^{2}\right)^{\top}\right)\left(\begin{array}{cc}
\varphi_{n}^{1} & 0 \\
0 & \varphi_{n}^{2}
\end{array}\right)\left(X_{n_{0}}\right)^{-1},
$$

since the $X_{n}$ 's are invertible by (5.5). Hence, by (5.4)-(5.6), there exist positive constants $M_{1}, M_{2}, M_{3}$ such that

$$
\begin{aligned}
\left\|\prod_{k=n_{0}}^{n} C_{k}\right\|^{2} & \leq M_{1} \max \left(\left|\varphi_{n}^{1}\right|^{2},\left|\varphi_{n}^{2}\right|^{2}\right)=M_{1}\left|\varphi_{n}^{1} \varphi_{n}^{2}\right| \max \left(\left|\frac{\varphi_{n}^{1}}{\varphi_{n}^{2}}\right|,\left|\frac{\varphi_{n}^{2}}{\varphi_{n}^{1}}\right|\right) \\
& \leq M_{2}\left|\varphi_{n}^{1} \varphi_{n}^{2}\right|=M_{2}\left|\operatorname{det}\left(\begin{array}{cc}
\varphi_{n}^{1} & 0 \\
0 & \varphi_{n}^{2}
\end{array}\right)\right| \\
& =M_{2}\left|\operatorname{det}\left(\left(y_{n+1}^{1}\right)^{\top},\left(y_{n+1}^{2}\right)^{\top}\right)\right|^{-1}\left|\operatorname{det} X_{n_{0}}\right| \prod_{k=n_{0}}^{n}\left|\operatorname{det} C_{k}\right| \\
& \leq M_{3} \prod_{k=n_{0}}^{n}\left|\operatorname{det} C_{k}\right| \mathbf{~}
\end{aligned}
$$

The above lemma allows us to combine the class $H$ with asymptotic discrete Levinson type results for equations of type (5.3) with $\left\{C_{n}\right\}_{n \geq n_{0}} \in$ $D^{k}$ (see [9]). Thus we are now ready to formulate and prove a new criterion.

CRITERION 5.10. Suppose that $C_{n}$ are invertible complex matrices for $n \geq n_{0}$, and that

$$
C_{n}=V_{n}+R_{n}, \quad n \geq n_{0},
$$

where $V_{n}$ are real matrices and

$$
\begin{gathered}
\left\{V_{n}\right\}_{n \geq n_{0}} \in D, \\
\limsup _{n \rightarrow \infty} \operatorname{discr} V_{n}<0, \\
\left\{R_{n}\right\}_{n \geq n_{0}} \in l^{1} .
\end{gathered}
$$

Then $\left\{C_{n}\right\}_{n \geq n_{0}} \in H$.

Proof. By [9, Th. 3.1] $\left({ }^{1}\right)$, there exists $n_{0}^{\prime} \geq n_{0}$ such that the equation

$$
x_{n+1}=C_{n} x_{n}, \quad n \geq n_{0}^{\prime},
$$

has two solutions $x^{1}, x^{2}$ of the form $x_{n}^{m}=\varphi_{n}^{m} y_{n}^{m}$ such that the scalar terms satisfy

$$
\varphi_{n}^{2}=\overline{\varphi_{n}^{1}} \neq 0
$$

$\left({ }^{1}\right)$ Note a misprint in the formulation of that theorem in [9]: the "Im" was omitted in the inequality $\inf _{n \geq N} \operatorname{Im} \lambda_{+}^{(k-1)}(n)>0$. 
and for the vector terms we have

$$
y_{n}^{m}=S_{n} \beta_{n}^{m}, \quad m=1,2,
$$

where $\beta_{n}^{m} \rightarrow e_{m}$, and

$$
\begin{aligned}
& \sup _{n \geq n_{0}^{\prime}}|| S_{n} \|<\infty, \\
& \inf _{n \geq n_{0}^{\prime}}\left|\operatorname{det} S_{n}\right|>0
\end{aligned}
$$

(the last two inequalities follow from the boundedness of $\left\{V_{n}\right\}_{n \geq n_{0}},(5.8)$ and from [9, Section 2.3.1]). Therefore by (5.10) and (5.11) we have $\left|\varphi_{n}^{1} / \varphi_{n}^{2}\right|=1$, and

$$
\left(\left(y_{n}^{1}\right)^{\top},\left(y_{n}^{2}\right)^{\top}\right)=S_{n} E_{n}, \quad E_{n}:=\left(\left(\beta_{n}^{1}\right)^{\top},\left(\beta_{n}^{2}\right)^{\top}\right) \rightarrow I .
$$

Thus, taking possibly $n_{0}^{\prime \prime} \geq n_{0}^{\prime}$, employing (5.12) and (5.13), we can use Lemma 5.9 to get $\left\{C_{n}\right\}_{n \geq n_{0}^{\prime \prime}} \in H$. This finishes the proof by Proposition $5.7(\mathrm{i})$.

In particular, taking $\left\{V_{n}\right\}_{n \geq n_{0}}$ convergent (which generally does not follow from the " $D$-assumption", in contrast to the $D^{1}$ special case) and $R_{n}=0$, we obtain the following result.

COROllary 5.11. If $C_{n}$ are real invertible matrices for $n \geq n_{0}$ and $\left\{C_{n}\right\}_{n \geq n_{0}}$ is a D sequence with $C_{n} \rightarrow C$ and $\operatorname{discr} C<0$, then $\left\{C_{n}\right\}_{n \geq n_{0}} \in H$.

Combining the above result with Theorem 5.6 and Proposition 5.7 we easily obtain the following convenient spectral result for $J$.

Fix $T \in \mathbb{N}$ and $l \in\{0,1, \ldots, T\}$, and for $\lambda \in \mathbb{C}$ and $n \geq 2$ set

$$
C_{n}(\lambda):=\prod_{s=0}^{T-1} B_{T n+s+l}(\lambda) .
$$

Corollary 5.12. Suppose that $J$ is self-adjoint, that (5.1) holds, and that

$$
\left\{\frac{w_{n-1}}{w_{n}}\right\}_{n \geq 2},\left\{\frac{w_{n}}{w_{n-1}}\right\}_{n \geq 2},\left\{\frac{1}{w_{n}}\right\}_{n \geq 1},\left\{\frac{q_{n}}{w_{n}}\right\}_{n \geq 1} \in l^{\infty} .
$$

If $G$ is an open subset of $\mathbb{R}$ and for any $\lambda \in G$,

(i) $\left\{C_{n}(\lambda)\right\}_{n \geq 2} \in D$,

(ii) $C_{n}(\lambda) \rightarrow C(\lambda)$ where $\operatorname{discr} C(\lambda)<0$,

then $(J)_{G} \sim \boldsymbol{X}_{G}, J$ is absolutely continuous in $G$ and $\bar{G} \subset \sigma_{\mathrm{ac}}(J)$.

5.3. $l^{2}$-solutions and pure pointedness. Let $J=J(w, q)$ be as in Section 5.2. We prove some results connecting the pure pointedness of $J$ with the existence of nontrivial $l^{2}$ generalized eigenvectors or solutions of some matrix equations given by "grouped" transfer matrices. 
We start from the following lemma concerning generalized eigenvectors (i.e., solutions of (1.2)). Note that this result is not as trivial as it seems to be at first sight, since we do not study eigenvectors, but generalized eigenvectors, i.e., the initial condition

$$
q_{1} u_{1}+w_{1} u_{2}=\lambda u_{1}
$$

need not be satisfied.

Lemma 5.13. Suppose that $J$ is self-adjoint and (5.1) holds. If $K \subset \mathbb{R}$ is such that for any $\lambda \in K$ there exists a nonzero generalized eigenvector for $J$ and $\lambda$, which belongs to $l^{2}$, then $J$ is pure point in $K$.

Proof. Let us study the subordinated solutions (briefly, s.s.) of (1.2) (see [12]). Define

$S_{0}:=\{\lambda \in \mathbb{R}:$ there exists a s.s. $u$ of (1.2) that does not satisfy (5.15) $\}$, $S_{\text {sing }}:=\{\lambda \in \mathbb{R}:$ there exists a s.s. $u$ of (1.2) and it satisfies (5.15) $\}$.

Any nonzero $l^{2}$ solution of (1.2) is a s.s. (see [12]). Hence, defining $K_{0}:=$ $K \cap S_{0}$ and $K_{1}:=K \cap S_{\text {sing }}$, we get $K=K_{0} \cup K_{1}$. Thus $\mathcal{H}_{K}(J)=\mathcal{H}_{K_{0}}(J)+$ $\mathcal{H}_{K_{1}}(J)$. But $\mathcal{H}_{K_{0}}(J) \subset \mathcal{H}_{S_{0}}(J)=\{0\}$, since $E_{S_{0}}(J)=0$ as follows from [12]. Therefore we have

$$
\mathcal{H}_{K}(J)=\mathcal{H}_{K_{1}}(J)
$$

But if $\lambda$ is in $K_{1}$, then (1.2) has a s.s. which satisfies (5.15) and it also has a nonzero $l^{2}$ solution $u$. So, $u$ is also a s.s., and since subordinated solutions are uniquely determined up to a scalar multiple, $u$ satisfies (5.15). Thus $u$ is an eigenvector for $J$ and $\lambda \in \sigma_{\mathrm{pp}}(J)$. In particular, $K_{1}$ is at most countable, and by Proposition 5.15 below, $\mathcal{H}_{K_{1}}(J) \subset \mathcal{H}_{\mathrm{pp}}(J)$. Hence $\mathcal{H}_{K}(J) \subset \mathcal{H}_{\mathrm{pp}}(J)$.

We now consider the equation

$$
x_{n+1}=C_{n}(\lambda) x_{n}, \quad n \geq n_{0},
$$

for a sequence $x=\left\{x_{n}\right\}_{n \geq n_{0}}$ of $\mathbb{C}^{2}$ vectors, where the matrices $C_{n}(\lambda)$ are "grouped" transfer matrices and $n_{0} \geq 2$, i.e., similarly to Section 5.2 , we fix $T \in \mathbb{N}, l \in\{0,1, \ldots, T\}$, and for $\lambda \in \mathbb{C}, n \geq 2$ we consider $C_{n}(\lambda)$ given by (5.14).

Observe that if $x=\left\{x_{n}\right\}_{n \geq n_{0}} \in l^{2}$ is a nonzero solution of (5.16) and the sequence of transfer matrices for $J$ and $\lambda$ is bounded, then there exists a scalar sequence $u$ which is a generalized eigenvector for $J$ and $\lambda$, which belongs to $l^{2}$ and is nonzero. For $m \geq T n_{0}+l$ the term $u_{m}$ is given by

$$
u_{m}=\left(\left(\prod_{s=p_{n}}^{m-1} B_{s}(\lambda)\right) x_{n}\right)_{2} \quad \text { for } p_{n} \leq m<p_{n+1} \text { and } n \geq n_{0},
$$

where $p_{n}=T n+l$ for $n \geq n_{0}$, and $y_{j}$ denotes the $j$ th coordinate of $y \in \mathbb{C}^{2}$ for $j=1,2$. Thus, by Lemma 5.13 we get the following result. 
Corollary 5.14. Suppose that $J$ is self-adjoint, (5.1) holds, and

$$
\left\{\frac{w_{n-1}}{w_{n}}\right\}_{n \geq 2},\left\{\frac{1}{w_{n}}\right\}_{n \geq 1},\left\{\frac{q_{n}}{w_{n}}\right\}_{n \geq 1} \in l^{\infty} .
$$

If $K \subset \mathbb{R}$ and for any $\lambda \in K$ there exists a nonzero solution of (5.16), which belongs to $l^{2}$, then $J$ is pure point in $K$.

5.4. Some general spectral facts. In Section 4 we have used two simple spectral results, valid for any self-adjoint operator in a separable Hilbert space. For the convenience of the reader, they are formulated explicitly here and short proofs are provided.

Proposition 5.15. Assume that $A$ is a self-adjoint operator in a separable Hilbert space.

(i) If $G$ is a Borel subset of $\mathbb{R}$, then $\sigma\left((A)_{G}\right) \subset \overline{G \cap \sigma(A)}$; in particular, $\sigma\left((A)_{G}\right) \subset \bar{G}$ (by convention, the empty set is the spectrum of the operator acting in the degenerate space $\{0\})$.

(ii) If $S \subset \mathbb{R}$ is at most countable, then $A$ is pure point in $S$.

Proof. (i) It can be easily checked that $E_{\omega}\left((A)_{G}\right)$ is the restriction of $E_{\omega}(A)$ to the space $\mathcal{H}_{G}(A)$ for any Borel set $\omega \subset \mathbb{R}$. Thus we have

$$
E_{\omega \cap G}(A)=0 \Rightarrow E_{\omega}(A) E_{G}(A)=0 \Rightarrow E_{\omega}\left((A)_{G}\right)=0 .
$$

We can apply the above for $\omega:=\mathbb{R} \backslash \overline{G \cap \sigma(A)}$, since then $\omega \cap G \subset \rho(A)$ (with $\rho$ denoting the resolvent set). But $\omega$ is an open set, so $E_{\omega}\left((A)_{G}\right)=0$ means that $\omega \subset \rho\left((A)_{G}\right)$, which proves (i).

(ii) We must prove that $\mathcal{H}_{S}(A) \subset \mathcal{H}_{\mathrm{pp}}(A)$, i.e., $E_{S}(A) v \in \mathcal{H}_{\mathrm{pp}}(A)$ for any vector $v$. This follows immediately from the strong countable additivity of the spectral projection measure and from the fact that for any $\lambda \in \mathbb{R}$, $E_{\{\lambda\}}(A) v$ is either 0 or an eigenvector of $A$, and thus it belongs to $\mathcal{H}_{\mathrm{pp}}(A)$.

Acknowledgements. This research was supported by the MSHE Grant N N 201426533 and the Intas Grant no. 05-1000008-7883.

\section{References}

[1] P. A. Cojuhari, Discrete spectrum in the gaps for perturbations of periodic Jacobi matrices, J. Comput. Appl. Math. (2008), doi: 10.1016/j.cam.2008.07.046.

[2] P. Deift and R. Killip, On the absolutely continuous spectrum of one-dimensional Schrödinger operators with square summable potentials, Comm. Math. Phys. 203 (1999), 341-347.

[3] J. Dombrowski and J. Nevai, Orthogonal polynomials, measures and recurrence relations, SIAM J. Math. Anal. 17 (1986), 752-759.

[4] L. Golinskii and P. Nevai, Szegö difference equations. Transfer matrices and orthogonal polynomials on the unit circle, Comm. Math. Phys. 223 (2001), 223-259. 
[5] G. Heinig, Inversion of periodic Jacobi matrices, Mat. Issled. 8 (1973), 180-200 (in Russian).

[6] H. Hochstadt, On the theory of Hill's matrices and related inverse spectral problems, Linear Algebra Appl. 11 (1975), 41-52.

[7] J. Janas and M. Moszyński, Alternative approaches to the absolute continuity of Jacobi matrices with monotonic weights, Integral Equations Operator Theory 43 (2002), 397-416.

[8] - - - Spectral properties of Jacobi matrices by asymptotic analysis, J. Approx. Theory 120 (2003), 309-336.

[9] - - - New discrete Levinson type asymptotics of solutions of linear systems, J. Difference Equations Appl. 12 (2006), 133-163.

[10] J. Janas and S. Naboko, Multithreshold spectral phase transitions for a class of Jacobi matrices, in: Oper. Theory Adv. Appl. 124, Birkhäuser, 2001, 267-285.

[11] —, - Jacobi matrices with power-like weights-grouping in blocks approach, J. Funct. Anal. 166 (1999), 218-243.

[12] S. Khan and D. B. Pearson, Subordinacy and spectral theory for infinite matrices, Helv. Phys. Acta 65 (1992), 505-527.

[13] R. Killip, Perturbations of one-dimensional Schrödinger operators preserving the absolutely continuous spectrum, Int. Math. Res. Notices 2002, no. 38, 2029-2061.

[14] A. Kiselev, Y. Last and B. Simon, Stability of singular spectral types under decaying perturbations, J. Funct. Anal. 198 (2003), 1-27.

[15] S. Kupin, Spectral properties of Jacobi matrices and sum rules of special form, J. Funct. Anal. 227 (2005), 1-29.

[16] A. Laptev, S. Naboko and O. Safronov, On new relations between spectral properties of Jacobi matrices and their coefficients, Comm. Math. Phys. 241 (2003), 91-110.

[17] Y. Last, Destruction of absolutely continuous spectrum by perturbation potentials of bounded variation, ibid. 274 (2007), 243-252.

[18] M. Moszyński, Spectral properties of some Jacobi matrices with double weights, J. Math. Anal. Appl. 280 (2003), 400-412.

[19] P. B. Naiman, On the spectral theory of non-symmetric periodic Jacobi matrices, Zap. Mekh.-Mat. Fak. Kharkov. Gos. Univ. i Kharkov. Mat. Obshch. 30 (1964), no. 4, 138-151 (in Russian).

[20] M. Reed and B. Simon, Methods of Modern Mathematical Physics, Vol. III, Academic Press, New York, 1979.

[21] C. Remling, The absolutely continuous spectrum of one-dimensional Schrödinger operators with decaying potentials, Comm. Math. Phys. 193 (1998), 151-170.

[22] G. Stolz, Spectral theory for slowly oscillating potentials. I. Jacobi matrices, Manuscripta Math. 84 (1994), 245-260.

[23] G. Teschl, Jacobi Operators and Completely Integrable Nonlinear Lattices, Amer. Math. Soc., Providence, 2000.

Wydział Matematyki, Informatyki i Mechaniki

Uniwersytet Warszawski

Banacha 2

02-097 Warszawa, Poland

E-mail: mmoszyns@mimuw.edu.pl

Received March 28, 2008

Revised version November 14, 2008 